\title{
The beam pattern of the IRAM 30-m telescope
}

\section{(a reflector with several surface error distributions)}

\author{
A. Greve ${ }^{1}$, C. Kramer ${ }^{2,3}$, and W. Wild ${ }^{2}$ \\ 1 IRAM, 300 rue de la Piscine, Domaine Universitaire, F-38406 St. Martin d'Hères, France \\ 2 IRAM, Nucleo Central, Avda. Divina Pastora 7, E-18012 Granada, Spain \\ 3 I. Physikalisches Institut, Universität zu Köln, Zülpicher Straße 77, D-50937 Köln, Germany
}

Received July 28, 1997; accepted June 11, 1998

\begin{abstract}
Total power scans across the Moon around New Moon (mostly day time) and Full Moon (night time) at $3.4 \mathrm{~mm}(88 \mathrm{GHz}), 2.0 \mathrm{~mm}(150 \mathrm{GHz}), 1.3 \mathrm{~mm}(230 \mathrm{GHz})$, and $0.86 \mathrm{~mm}(350 \mathrm{GHz})$ wavelength are used to derive the beam pattern of the IRAM 30-m telescope to a level of approximately $-30 \mathrm{~dB}(0.1 \%)$ and, dependent on wavelength, to a full width of $1000-1400^{\prime \prime}$. From the reflector surface construction and application of the antenna tolerance theory we find that the measurable beam consists of the diffracted beam, two underlying error beams which can be explained from the panel dimensions, and a beam deformation mostly due to large-scale transient residual thermal deformations of the telescope structure. In view of the multiple beam structure of the $30-\mathrm{m}$ telescope, and of other telescopes with a similar reflector construction of (mini-)panels and panel frames, we summarize the antenna tolerance theory for the influence of several independent surface/wavefront deformations. This theory makes use of different correlation lengths, which in essence determine the independent error distributions, and of the wavelength-scaling of the diffracted beam and of the error beams.
\end{abstract}

From the Moon scans we derive the parameters for calculation of the $30-\mathrm{m}$ telescope beam in the wavelength range $3 \mathrm{~mm}$ to $0.8 \mathrm{~mm}$ as required for the reduction of astronomical observations, in particular of extended sources. The parameters of the beam are primarily for the time after July 1997 when the reflector was re-adjusted and improved to the illumination weighted surface precision of $\sigma_{\mathrm{T}}=0.065-0.075 \mathrm{~mm}$.

In the Appendix we explain the choice for this analysis of scans taken around New Moon and Full Moon.

Key words: telescopes

Send offprint requests to: A. Greve

\section{Introduction}

Observations with a single dish radio telescope are made with a finite size beam of which the theoretical pattern is often degraded by imperfections of the telescope, primarily deformations of the main reflector surface. A knowledge of the actual beam profile is required for the reduction of astronomical observations and estimates of a possible improvement of the telescope. The theoretical beam pattern can be calculated from the Theory of Physical Optics (Born \& Wolf 1980; Love 1978; Christiansen \& Högbom 1985; Kraus 1986); the beam degradation can be calculated from the Theory of Aberrations (Born \& Wolf 1980) and the Antenna Tolerance Theory (Scheffler 1962; Ruze 1952, 1966; Shifrin 1971; Baars 1973), both specified by a few basic parameters which must be determined from measurements of the wavefront error topography or the beam pattern itself. The wavefront (reflector surface) error topography can be derived, for instance, from holography measurements (Morris et al. 1988; Whyborn \& Morris 1995); the actual beam pattern can also be derived, for instance, from scans across a strong point-like radio source or a satellite beacon, or from scans across the limb of the Moon and the Sun (Horne et al. 1981; Lindsey \& Roellig 1991). The measured beam pattern reveals, in general, the influence of spatially large--scale and small-scale wavefront deformations. Large-scale deformations distort the central part of the beam; small-scale deformations produce one, or several, underlying, extended error beams. We analyze total power scans across the Moon at $3.4 \mathrm{~mm}$ $(88 \mathrm{GHz}), 2.0 \mathrm{~mm}(150 \mathrm{GHz}), 1.3 \mathrm{~mm}(230 \mathrm{GHz})$, and $0.86 \mathrm{~mm}(350 \mathrm{GHz})$ wavelength, and provide in addition to the earlier investigation of Garcia-Burillo et al. (1993) the parameters of an analytic expression of the IRAM $30-\mathrm{m}$ telescope beam as required for the reduction of astronomical observations, in particular of extended sources. (For a description of the $30-\mathrm{m}$ telescope and its behaviour see 
Baars et al. $(1987,1994)$ and Greve et al. $(1993,1996 a$, 1998)).

This publication consists of two parts. The first part explains the theory of beam degradation from several surface error distributions, and we confirm this theory with multi-wavelength beam patterns of the $30-\mathrm{m}$ telescope derived from Moon limb scans observed before July 1997 (Sects. 2,3). The second part explains the result of the latest surface adjustment (July 1997), and we provide the current parameters for calculation of the $30-\mathrm{m}$ telescope beam (Sects. 4-6). In detail, Sect. 2 summarizes the antenna tolerance theory for a combination of several largescale and small-scale wavefront (reflector surface) deformations, as appropriate for the understanding of the $30-\mathrm{m}$ telescope and other telescopes of similar reflector design. In this theory we use the deformation correlation length(s) to anticipate the structure of the degraded beam from details of the reflector surface construction. We explain in Sects. 3.1-3.3 how we derive in an empirical way the parameters of the degraded beam from the comparison of observed and calculated scans across the limb of the Moon, taken around New Moon (mostly day time) and Full Moon (night time). In particular we confirm the wavelength scaling of the error beam(s). In Sect. 3.4 we show the reflector surface error correlation function, derived from holography measurements, which confirms in an independent way the correlation lengths used in the analysis of the Moon scans. We explain in Sect. 3.5 in which way the standard Ruze relation is modified for the case of several error distributions. In Sect. 4 we explain the surface precision obtained from the July 1997 panel frame adjustment (Morris et al. 1996, 1997). Sect. 5 shows the current beam patterns of the $30-\mathrm{m}$ telescope at $3.4 \mathrm{~mm}, 2.0 \mathrm{~mm}$, and $1.3 \mathrm{~mm}$, and Sect. 6 gives the current telescope efficiencies. In the Appendix we explain our choice of scans around New Moon and Full Moon. We follow the notation used by Downes (1989) and used at the 30-m telescope (see Mauersberger et al. 1989).

\section{Terminology and theory}

\subsection{The structure of the beam pattern}

The directional response, i.e. the beam $\mathcal{F}=\mathcal{F}_{\mathrm{c}}+\mathcal{F}_{\mathrm{e}}$ of a single dish radio telescope consists of the diffracted beam $\mathcal{F}_{\mathrm{c}}$, formed by coherently focused radiation, and the error beam $(\mathrm{s}) \mathcal{F}_{\mathrm{e}}$, formed by radiation "scattered" towards the focal plane. The diffracted beam $\mathcal{F}_{\mathrm{c}}=\mathcal{F}_{\mathrm{mb}}+\mathcal{F}_{\text {sl }}$ consists of the main beam $\mathcal{F}_{\text {mb }}$ and sidelobes $\mathcal{F}_{\text {sl }}$. The diffracted beam of a perfect, circular reflector of diameter $D$ (with vertex hole, shadowing from the subreflector and its support, illumination taper of the receiver etc.) is a tapered Airy-type pattern $\mathcal{A}_{\mathrm{T}}$ with components $\mathcal{A}_{\mathrm{T}}=\mathcal{A}_{\mathrm{T}, \mathrm{mb}}+$ $\mathcal{A}_{\mathrm{T}, \mathrm{sl}}$. For a shallow, perfect, full aperture, non-tapered reflector the diffracted beam is $\mathcal{A}(u)=\left[J_{1}(u) / u\right]^{2}$ with $J_{1}$ the Bessel function of first order and $u$ the spatial coordinate of the focal plane (Born \& Wolf 1980). The steepness of a radio reflector and the illumination taper preserves to a large extent the sidelobe structure of the Airy pattern (Minnett \& Thomas 1968; Goldsmith 1987; see Sect. 5), however, the sidelobe levels are made by purpose significantly lower than those of the non-tapered beam. The width (FWHP) of the main beam $\mathcal{A}_{\mathrm{T}, \mathrm{mb}}$ is

$\theta_{\mathrm{b}}=k \lambda / D \quad[\mathrm{rad}]$

with $k(1 \leq k \lesssim 1.4)$ a factor dependent on the illumination taper and blockage of the aperture (Christiansen \& Högbom 1985; Kraus 1986). Measurements with the 30-m telescope and current SIS receivers of $\sim-13 \mathrm{~dB}$ Gaussian edge taper give $k=1.16$ for $0.8 \mathrm{~mm} \lesssim \lambda \lesssim 3 \mathrm{~mm}$ (Kramer 1997).

We assume in the following that the beam is degraded by phase perturbations of the wavefront $\delta_{\varphi} \approx 2(2 \pi / \lambda) \delta$ which are primarily due to deformations $\delta$ of the main reflector surface. For a good quality telescope we may assume also that the phase perturbations are small compared to the wavelength so that the resulting beam degradation is the sum of the individual degradations (see Shifrin 1971; Sect. 2.4). In addition we assume that the main reflector surface is constructed from a large number of panels. Large-scale surface deformations, which do not change significantly over several panel areas or a considerable fraction of the reflector surface, degrade the diffraction pattern but preserve, in general, the main beam and sidelobe structure. Small-scale wavefront deformations, which change significantly over single panel areas or panel sub-sections, produce the underlying error beams. Surface deformations which change over distances of wavelengths behave like rough surfaces, and are discussed in optical journals.

Different mathematical formalisms are used to calculate the beam degradation from spatially large-scale and small-scale wavefront deformations.

\subsection{Large-scale deformations}

Large-scale deformations, which often appear as systematic deformations, are described by combinations of low order Zernike polynomials $Z_{i j}(i \lesssim 10, j \lesssim 10)$

$\delta=\sum_{i, j} a_{i j} Z_{i j}=\sum_{i, j} a_{i j} R_{i}(\rho) \cos (j \psi)$

(with $R_{i}$ functions of the normalized aperture radius $\rho$, $\psi$ the azimuth angle of the aperture, and $a_{i j}$ the amplitude of component $(i j)$ ) (Born \& Wolf 1980; Greve et al. 1996b), or other orthogonal functions for decomposition of wavefront deformations (Smith \& Bastian 1997). The corresponding, degraded, tapered beam pattern $\mathcal{Z}_{\mathrm{c}, \mathrm{T}}$ has a main beam and sidelobes, and can be calculated exactly from diffraction theory. Well known examples are 
defocus $\left(\mathcal{Z}_{10}, \mathcal{Z}_{20}\right)$, coma $\left(\mathcal{Z}_{31}\right)$, and astigmatism $\left(\mathcal{Z}_{22}\right)$. These deformations are sometimes due to misaligned components, for instance a misaligned subreflector or a misaligned receiver.

\subsection{Small-scale deformations}

Small-scale random ${ }^{1}$ deformations of the reflector surface are characterized by the (root mean square) rms-value $\sigma$ and the spatial correlation function $C_{\delta}(d)(0 \leq d \leq$ $D)^{2}$. For a reflector surface sampled at a large number of positions ( $n=1,2, \ldots, K$; with $K$ a few hundred), the rms-value is

$\sigma^{2}=\sum_{n=1, K}\left(\delta_{n}\right)^{2} / K, \quad \sum \delta_{n}=0$

for deformations $\delta_{n}$ measured in the normal direction of the best-fit reflector surface. The quantity $\sigma$ is the geometrical rms-value of the surface errors (Greve \& Hooghoudt 1981). The rms-value $\sigma_{\varphi}$ of the associated wavefront deformation (including a factor 2 because of reflection) is

$\sigma_{\varphi}=R(4 \pi / \lambda) \sigma=2 R k \sigma=2 k \sigma_{\mathrm{T}}, \quad k=2 \pi / \lambda$

with the reduction factor $R=0.8-0.9$ for a steep radio reflector of focal ratio $N=F / D \approx 0.3$ and $a-10 \mathrm{~dB}$ to $-15 \mathrm{~dB}$ edge taper of the illumination. $\sigma_{\mathrm{T}}=\mathrm{R} \sigma$ is the tapered, or illumination weighted, rms-value of the surface errors (Greve \& Hooghoudt 1981) as derived, for instance, from aperture efficiency and holography measurements. The normalized correlation function $C_{\delta}(d)$ with $C_{\delta}(0)=1$ is

$C_{\delta}(d)=\left[\sum_{(1,2)} \delta\left(\mathbf{r}_{1}\right) \delta\left(\mathbf{r}_{2}\right) / N_{1,2}\right] / \sigma^{2}=C_{\delta}^{+} / \sigma^{2}$

with the summation $(1,2)$ extending over the number $N_{1,2}$ of pairs $\left(\mathbf{r}_{1}, \mathbf{r}_{2}\right)[\mathbf{r}=(x, y)]$ of positions in the aperture (A) with separation

$\left|\mathbf{r}_{1}-\mathbf{r}_{2}\right|=d \leq D$.

The correlation function of the corresponding wavefront deformations $\left[\delta_{\varphi}\right]$ is

$C_{\varphi}(d)=\left[\sum_{(1,2)} \delta_{\varphi}\left(\mathbf{r}_{1}\right) \delta_{\varphi}\left(\mathbf{r}_{2}\right) / N_{1,2}\right] / \sigma_{\varphi}^{2}=C_{\varphi}^{+} / \sigma_{\varphi}^{2}$

with $C_{\varphi} \approx C_{\delta}$. The degraded beam pattern at the position $\mathbf{u}=(u, v)$ of the focal plane is (Scheffler 1962)

$\mathcal{F}(\theta) \equiv \mathcal{F}(\mathbf{u}) \propto$

\footnotetext{
1 We exclude small-scale periodic deformations which act like gratings (for instance warped panels, regular surface ripples from machining, etc.), although such deformations occasionally do occur. The grating theory of a paneled reflector surface is not available; however, some special investigations have been published (Cortes-Medellin \& Goldsmith 1994; Hills \& Richer 1992; Harris et al. 1997).

${ }^{2}$ The values $\sigma$ and $C_{\delta}$ can also be calculated for large-scale deformations, however, they do not always contain a physical meaning as for small-scale random deformations (Greve 1980).
}

$\exp \left[-\left(\sigma_{\varphi}\right)^{2}\right] \iint_{A} \mathrm{~d} S_{1} \mathrm{~d} S_{2} \exp \left[C_{\varphi}^{+}(d)\right] \exp \left[i k \mathbf{u}\left(\mathbf{r}_{1}-\mathbf{r}_{2}\right) / f\right](8)$ with $\mathrm{d} S_{\mathrm{i}}=(\mathrm{d} x \mathrm{~d} y)_{i}$ a surface element of the aperture $(\mathrm{A})$. The angular distance $\theta$ from the focal axis of the position $\mathbf{u}=(u, v)$ is $\theta=\sqrt{u^{2}+v^{2}} / f$ [rad], with $f$ the effective focal length of the telescope. In order to obtain an analytic expression of Eq. (8), it has been assumed (Scheffler 1962; Ruze 1966; Shifrin 1971; see the criticism/support by Schwesinger 1972; Greve et al. 1994a) that ${ }^{3}$

$C_{\delta}(d) \approx C_{\varphi}(d) \propto \exp \left[-(d / L)^{2}\right]$

with $L$ the correlation length ${ }^{4}$ of the deformations $[\delta]$. Under the assumption of a Gaussian correlation length distribution (Eq. (9)), the degraded beam pattern $\mathcal{F}(\theta)$ is circular symmetric and

$\mathcal{F}(\theta)=\mathcal{F}_{\mathrm{c}}(\theta)+\mathcal{F}_{\mathrm{e}}(\theta)$

$=\exp \left[-\left(\sigma_{\varphi}\right)^{2}\right] \mathcal{A}_{\mathrm{T}}(\theta)+a_{\mathrm{e}} \exp \left[-(\pi \theta L / 2 \lambda)^{2}\right]$

with

$a_{\mathrm{e}}=(L / D)^{2}\left[1-\exp \left[-\left(\sigma_{\varphi}\right)^{2}\right]\right] / \epsilon_{0}$

for $\sigma_{\varphi} \lesssim 1$ (Scheffler 1962; Ruze 1966; Vu 1969; Baars 1973) as existing on a good quality telescope. $\epsilon_{0}$ is the aperture efficiency at long wavelengths. The normalization is $\mathcal{F}(\theta=0)=1$ for $\sigma=0$ (which implies $L=0)$. The width (FWHP) of the error beam is

$\theta_{\mathrm{e}}=0.53 \lambda /(L / 2) \quad[\mathrm{rad}]$.

This tolerance theory of a single small-scale error distribution $[\delta]$ with correlation length $L$, as discussed in the basic publication of Scheffler (1962), Ruze (1966), and Robieux (1966), is verified by a large number of investigations of optical telescopes and radio telescopes (see in particular Ruze 1966; Vu 1970a) ${ }^{5}$.

\subsection{The combination of small-scale and large-scale surface deformations}

It is reasonable to assume that a paneled reflector surface may have several independent error distributions $\left[\delta_{i}\right]$ with

\footnotetext{
${ }^{3}$ Shifrin (1971) analyzes also the exponential correlation length distribution; however, arguments are given that the Gaussian correlation length distribution represents the more realistic case.

${ }^{4} L=2$ c, with c the correlation radius (Ruze 1966; Baars 1973).

5 The term $\mathcal{F}_{\mathrm{c}}(\theta)$ of Eq. (10) was first derived by Väisälä (1922) to quantify the precision of optical surfaces. The term was re-discovered by Marechal (1947), Scheffler (1962), Ruze (1966), Robieux (1966), and others, however, with the understanding of being valid only in the case of an uncorrelated error distribution $(L=0)$. Nevertheless, this term is frequently used in efficiency calculations although, apriori, being correct only in case of uncorrelated errors (see Sect. 3.5).

The derivation of the beam pattern Eq. (8) is valid for a shallow reflector as used in optical telescopes. However, Eq. (10) can also be used for a steep radio reflector in case the appropriate diffraction pattern $\mathcal{A}_{\mathrm{T}}$ is used (see Sect. 5).
} 
different rms-values $\left[\sigma_{i}\right]$ and different correlation lengths $\left[L_{i}\right]$. The surface of the IRAM $30-$ m reflector $^{6}$ contains independent large-scale and small-scale errors of which the characteristic correlation lengths are anticipated from the mechanical construction.

The surface of the 30-m reflector consists of 7 rings of panel frames (in total 210) with each frame holding two panels. A panel (average size $\sim 1 \times 2$ meter) is attached to its frame by 15 screws, arranged in 5 parallel rows with approximately $1 / 4 \times 2 \mathrm{~m} \approx 0.5 \mathrm{~m}$ spacing. These support screws were used to adjust the panel contours to an average precision $\sigma_{\mathrm{p}} \approx 0.03 \mathrm{~mm}$, as measured in the factory (Baars et al. 1987). From the geometry of the panel support and contour maps of the panel surfaces (Fig. 1) as measured in the factory, we anticipate that the residual deformations of the adjustments have a correlation length $L_{\mathrm{p}}$ of approximately $1 / 4$ length of a panel, so that $L_{\mathrm{p}}=0.3-0.5 \mathrm{~m}$ and $L_{\mathrm{p}} / D \approx 1 / 75$. The width of the anticipated error beam is $\theta_{\mathrm{e}, \mathrm{p}} \approx 75 \theta_{\mathrm{b}}$ (Eqs. $\left.(1,12)\right)$.

A panel frame (average size $\sim 2 \times 2$ meter) is attached to the backstructure by adjustment screws located at the four frame corners. A panel frame which is misaligned in piston and/or tilt represents a surface area of correlated deformations. The weighted distance between the centers of adjacent panel frames gives the correlation length $L_{\mathrm{a}}=1.5-2.0 \mathrm{~m}$ so that $L_{\mathrm{a}} / D \approx 1 / 17$. The width of the anticipated error beam is $\theta_{\mathrm{e}, \mathrm{a}} \approx 17 \theta_{\mathrm{b}}$ (Eqs. $\left.(1,12)\right)$.

For two independent small-scale surface error distributions $\left[\delta_{1}\right]$ and $\left[\delta_{2}\right]$, with Gaussian correlation length distributions $L_{1}$ and $L_{2}$, the effective surface rms-value $\sigma$ is (see Eq. (3))

$\sigma^{2}=\sum\left(\delta_{1, n}+\delta_{2, n}\right)^{2} / K$

$=\sum\left(\delta_{1, n}\right)^{2} / \mathrm{K}+\sum\left(\delta_{2, n}\right)^{2} / K=\sigma_{1}^{2}+\sigma_{2}^{2}$

since $\sum \delta_{1, n} \delta_{2, n}=0$. The correlation function of the combined error distribution $[\delta]=\left[\delta_{1}\right]+\left[\delta_{2}\right]$ is

$C_{\delta}(d)=\left[C_{\delta, 1}^{+}(d)+C_{\delta, 2}^{+}(d)\right] /\left[\sigma_{1}^{2}+\sigma_{2}^{2}\right]$

$\propto \sum_{i=1,2} \exp \left[-\left(d / L_{i}\right)^{2}\right] / \sum \sigma_{i}^{2}$.

When using the correlation function of the corresponding wavefront deformation (see Eq. (7)) in Eq. (8) under the assumption that the phase rms-values $\sigma_{\varphi, i}$ are small so that the integral can be split into the sums of the individual contributions, i.e.

$\mathcal{F}(\theta)=\exp \left[-\sum_{i}\left(\sigma_{\varphi, i}\right)^{2}\right] \iint \mathrm{d} S_{1} \mathrm{~d} S_{2} \exp \left[\sum_{i} C_{\varphi, i}^{+}(d)\right] \exp [\ldots]$

\footnotetext{
${ }^{6}$ The subreflector of the 30-m Cassegrain telescope and the Nasmyth mirrors may have additional surface errors. However, in general, these mirrors are more precise than the main reflector, and thus may be neglected (see Rush \& Wohlleben 1982). For other reflectors with several error distributions see, for instance, the Itapetinga 14-m telescope (Kaufmann et al. 1987) and the JCMT 15-m telescope (Hills \& Richer 1992; Prestage 1993).
}
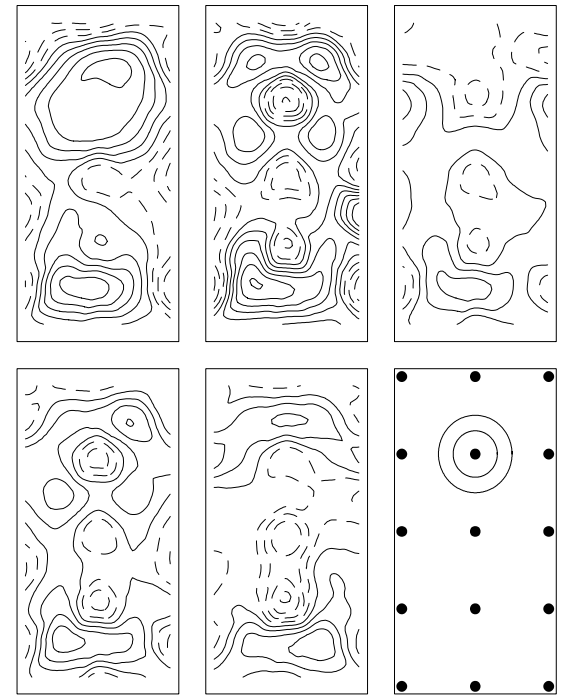

Fig. 1. Surface contours as measured in the factory of five randomly selected panels. The dots show the positions of the adjustment screws; the circles illustrate the size of a correlation cell of $L_{\mathrm{p}}=0.3-0.5 \mathrm{~m}$ diameter as the influence area of an adjustment screw. Contour levels at $0.015 \mathrm{~mm}$

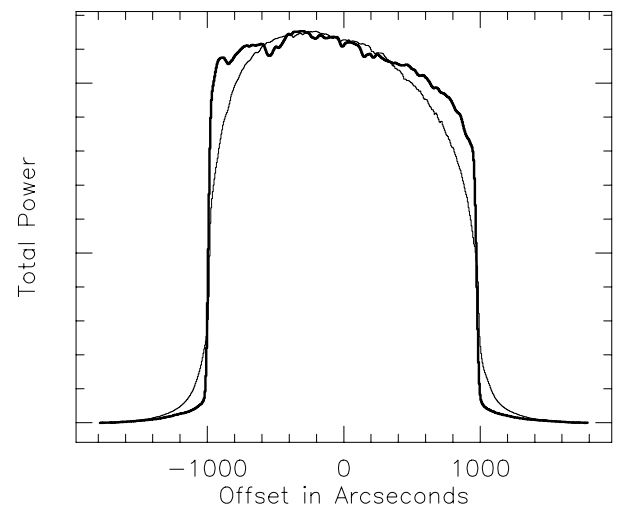

Fig. 2. Total power scans across the Moon. The $3.4 \mathrm{~mm}$ observation (thick line) was made 0.5 days after New Moon (6 Sep. 1994); the $0.86 \mathrm{~mm}$ observation (thin line) was made 4 days before New Moon (5 Mar. 1997). Note the decrease in sharpness of the limb with decreasing wavelength. (The structure of the $3.4 \mathrm{~mm}$ scan shows features of the Moon's surface)

$$
\approx \sum_{i} \exp \left[-\left(\sigma_{\varphi, i}\right)^{2}\right] \iint \mathrm{d} S_{1} \mathrm{~d} S_{2} \exp \left[C_{\varphi, i}^{+}(d)\right] \exp [\ldots]
$$

then the beam pattern degraded by several independent small-scale deformations $\left[\delta_{i}\right]$ with Gaussian correlation length distributions $\left[L_{i}\right]$ (Eq. (14)) is

$$
\mathcal{F}(\theta)=\exp \left[-\left(\sigma_{\varphi}\right)^{2}\right] \mathcal{A}_{\mathrm{T}}(\theta)+\sum_{i} a_{\mathrm{e}, i} \exp \left[-\left(\pi \theta L_{i} / 2 \lambda\right)^{2}\right](16)
$$

where the amplitude $a_{\mathrm{e}, i}=\left(L_{i} / D\right)^{2}\left[1-\exp \left[-\left(\sigma_{\varphi, i}\right)^{2}\right]\right] / \epsilon_{0}$ (Eq. (11)) of the error beam $[i]$ is related only to the rms-value $\sigma_{i}$ and the correlation length $L_{i}$ of the error distribution $\left[\delta_{i}\right]$. Equivalent to Eq. (13), in Eq. (16) $\sigma_{\varphi}^{2}=\sum\left(\sigma_{\varphi, i}\right)^{2}$. For a rigorous discussion of several small-scale error distributions, the proofs of Eq. (14) and 


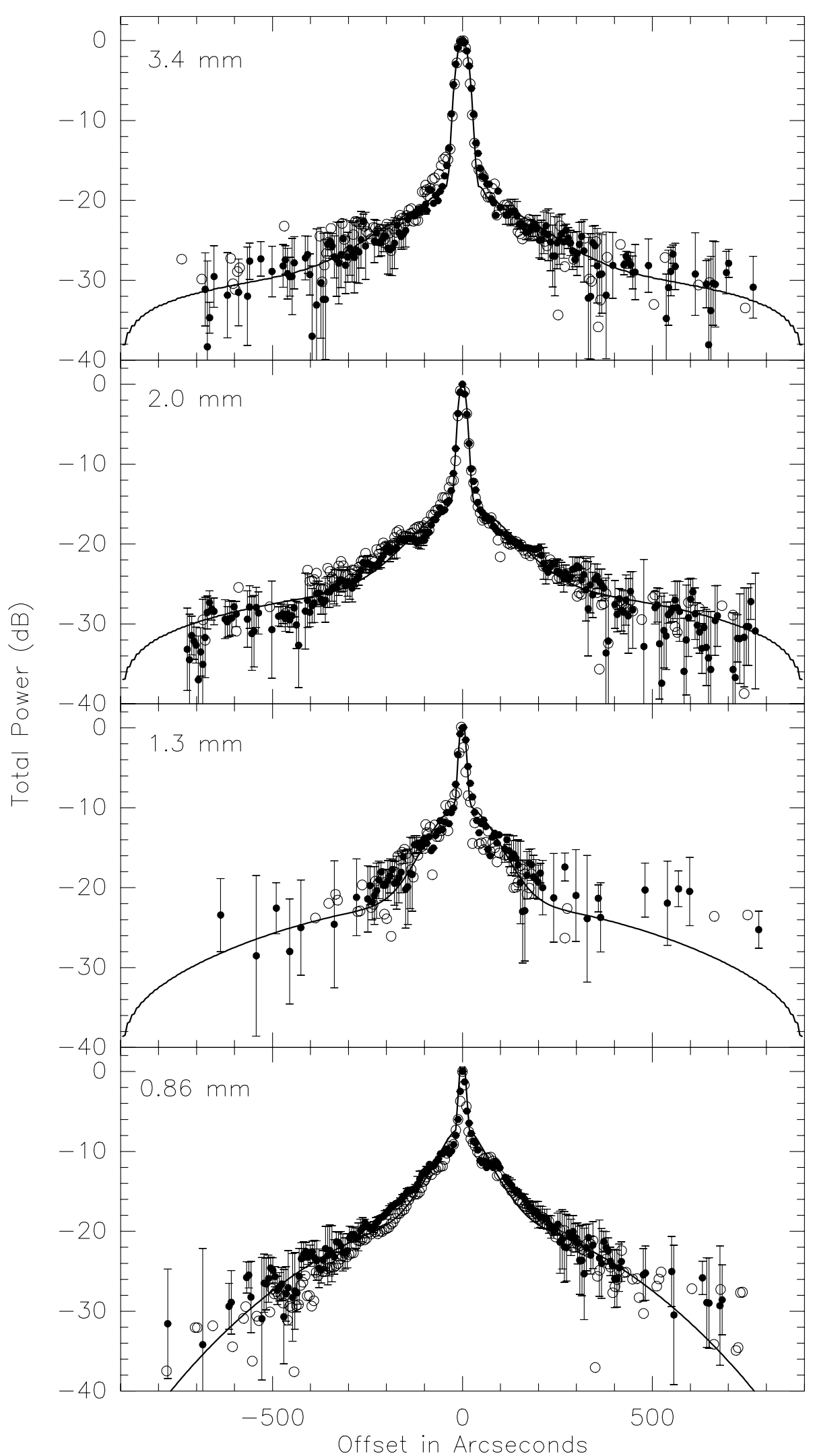

Fig. 3. Composite profiles $\left[f_{\mathrm{M}}(u)\right]$ (i.e. scans across the left and right limb of the Moon and differentiated along the scan direction) observed around New Moon. For each wavelength we show two measurements (solid dots, open circles) and error bars to demonstrate the repeatability of the observations. The solid lines show the synthetic best-fit profiles $\left[f_{\mathrm{S}}(u)\right]$, extrapolated somewhat beyond the measurements. The observations at $3.4 \mathrm{~mm}, 2 \mathrm{~mm}$, and $1.3 \mathrm{~mm}$ are made simultaneously; at $0.86 \mathrm{~mm}$ wavelength only observations 4 days before New Moon are available. (The data refer to the situation before July 1997) 
Eq. (16), and the validity of relation (15) see Shifrin (1971, chapter 5).

Following the explanation of Sect. 2.2, additional large-scale deformations cover areas of several panel frames so that their correlation length is, say, $L_{1} \approx 5 L_{\mathrm{a}}$. The width of the anticipated error beam is $\theta_{\mathrm{e}, 1} \approx 5 \theta_{\mathrm{b}}$ (Eqs. $(1,12))$. For large-scale deformations the diffraction pattern $\mathcal{A}_{\mathrm{T}}$ of Eq. (16) is replaced by the corresponding low order Zernike polynomial diffraction pattern $\mathcal{Z}_{\mathrm{c}, \mathrm{T}}$, for instance a comatic or astigmatic beam.

\section{The IRAM 30-m telescope beam structure}

We analyze multi-wavelength beam profiles of the $30-\mathrm{m}$ telescope obtained from Moon limb scans, and demonstrate that the antenna tolerance theory of several error distributions explained above gives consistent results for the beam structure and the efficiencies.

\subsection{Measurements}

We applied the method of total power Moon limb scans to derive the beam at $3.4 \mathrm{~mm}, 2 \mathrm{~mm}, 1.3 \mathrm{~mm}$, and $0.86 \mathrm{~mm}$ wavelength, using SIS receivers of $500 \mathrm{MHz}$ bandwith and similar Gaussian illumination of $\sim-13 \mathrm{~dB}$ edge taper. The scans of $3600^{\prime \prime}$ length, across the Moon of $\sim 1800^{\prime \prime}$ diameter, allow an investigation of the beam to a distance of $\sim \pm 900^{\prime \prime}$ from the beam axis. After focusing the telescope, the data of a scan were taken on-the-fly in $120 \mathrm{~s}$ at a spatial resolution of $6^{\prime \prime}$ (200 ms sampling rate). Linear baselines, determined at the outermost $\pm 30^{\prime \prime}$, were subtracted from the scans. The observations were made under very stable atmospheric conditions so that the measurements are reliable to a level of approximately $-30 \mathrm{~dB}$, i.e. $\sim 0.1 \%$ of the peak intensity ${ }^{7}$. The measurements were made at intermediate elevations where the homology deformations of the reflector are small (Greve et al. 1998). For the analysis we have taken scans around New Moon (mostly day time) and Full Moon (night time) which provide for the analysis the advantage of a simple brightness distribution of the Moon (Sect. 3.2, Appendix), and also the possibility to investigate the day and night time performance of the telescope.

Figure 2 shows a $3.4 \mathrm{~mm}$ and a $0.86 \mathrm{~mm}$ total power scan across the Moon, taken around New Moon. The distortion of the intrinsically sharp limb is due to the finite beam of the telescope.

\subsection{Moon scans and profiles}

Within the limitations of the antenna tolerance theory, the beam pattern (Eq. (16)) is circular symmetric so that any

\footnotetext{
7 Except the measurements at $1.3 \mathrm{~mm}$ wavelength (Fig. 3) made with a noisier receiver.
}

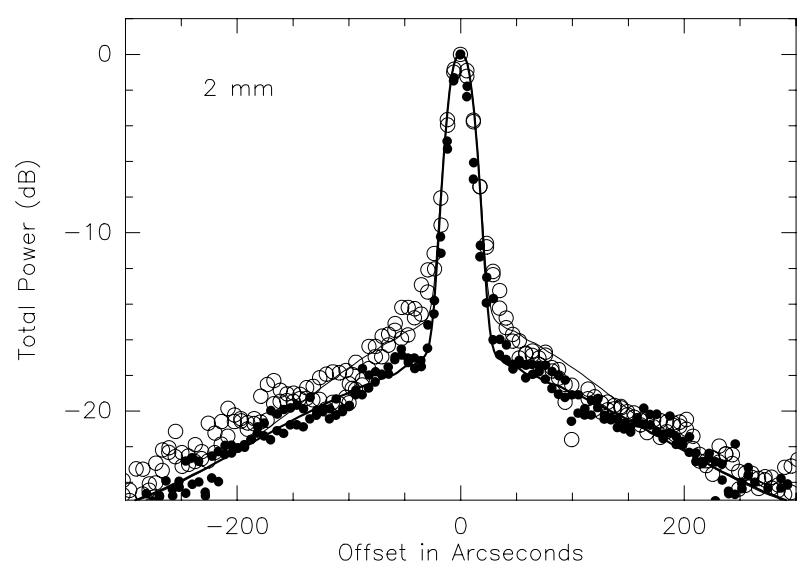

Fig. 4. Composite profiles $f_{\mathrm{M}}(u)$ derived from observations at $2 \mathrm{~mm}$ wavelength at Full Moon (dots) and New Moon (open circles, see Fig. 3). The heavy line and the thin line show the best-fit synthetic profile $f_{\mathrm{S}}(u)$ for Full Moon (night time) and New Moon (day time), respectively. The additional degradation during day time (New Moon) is a partially transient effect and is in many cases smaller than shown here. (The data refer to the situation before July 1997)

scan direction across the Moon, around New Moon and Full Moon, may be used to derive the beam parameters. For this we use an empirical approach and compare observed scans across the limb $\left[P_{\mathrm{M}}(u)\right.$, differentiated in scan direction called $\left.f_{\mathrm{M}}(u)\right]$ with synthetic scans $\left[P_{\mathrm{S}}(u)\right.$, differentiated in scan direction called $f_{\mathrm{S}}(u)$ ]. The synthetic scans are calculated from the convolution of the beam $\mathcal{F}(\theta) \equiv \mathcal{F}(u, v)$ (Eq. (16)) and the brightness distribution $T_{\mathrm{M}}(u, v)$ of the Moon. For measurements around New Moon and Full Moon we use the symmetric brightness distribution $T_{\mathrm{M}}=T_{0} \Pi(u, v)$ with $T_{0}$ the average brightness and

$\Pi(u, v)=1+C_{\mathrm{M}}\left(1-\rho^{2}\right), \rho=\sqrt{\left(u^{2}+v^{2}\right)} /\left(15^{\prime}\right)$

for $u^{2}+v^{2} \leq\left(15^{\prime}\right)^{2}$, and zero outside. For New Moon we use $C_{\mathrm{M}}=0$, for Full Moon we use $C_{\mathrm{M}}=0.5$ (see Fig. 10a below). With $u$ measured near culmination approximately in East-West direction through the center of the Moon, and $v$ perpendicular to this direction, a synthetic total power scan is

$P_{\mathrm{S}}(\mathrm{u}) \propto T_{0} \iint \mathcal{F}\left(u^{\prime}, v^{\prime}\right) \Pi\left(u-u^{\prime}, v^{\prime}\right) \mathrm{d} u^{\prime} \mathrm{d} v^{\prime}$.

In a similar way as applied to the observed profiles $f_{\mathrm{M}}(u)$, the synthetic composite profiles

$f_{\mathrm{S}}(u)=\mathrm{d} P_{\mathrm{S}}(u) / \mathrm{d} u$

are constructed from sections $f_{\mathrm{S}}(u)$ with the left and right wing of the profile taken outside $\left(15^{\prime} \leq|u|\right)$ the right and left limb of the Moon. For observations around New Moon and Full Moon, the sections $f_{\mathrm{S}}(u)$ of the left $(\sim$ East) and right $(\sim$ West $)$ wing are symmetric and may be added to obtain a composite profile (see Figs. 10b,c below). Figure 3 shows for New Moon the observed and 


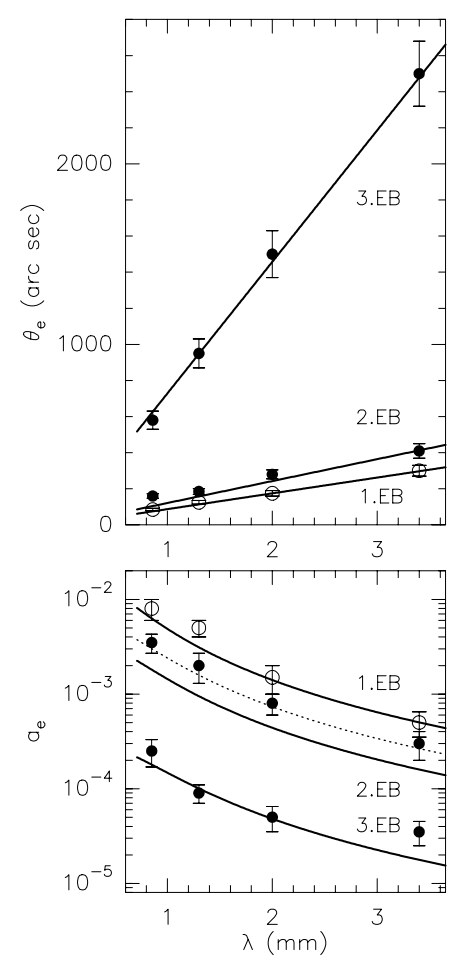

Fig. 5. Best-fit values (solid dots) of the widths $\theta_{\mathrm{e}}$ and amplitudes $a_{\mathrm{e}}$ of the error beams deduced from the observed profiles of Fig. 3, and their approximations (solid lines) from Eq. (12) and Eq. (11), respectively. EB denotes the different error beams. The 1st EB is a partially transient phenomenon; the data (open circles) are shown for New Moon day time measurements. The heavy lines are the current parameters of the beam pattern. The dashed line (2nd EB) and the related measurements (solid dots) refer to the situation before the adjustment of July 1997

the best-fit synthetic profiles, $f_{\mathrm{M}}(u)$ and $f_{\mathrm{S}}(u)$, respectively; Fig. 4 shows a similar profile at $2 \mathrm{~mm}$ wavelength observed at Full Moon. We emphasize that the differentiation (Eq. (19)) is not a deconvolution so that the profiles $f_{\mathrm{M}}$ and $f_{\mathrm{S}}$ shown in Fig. 3 and Fig. 4 do not exactly represent the true beam pattern of the telescope.

\subsection{The beam parameters}

We determined the best-fit beam pattern $\mathcal{F}(u, v)$ (Eq. (16)) in an empirical way by minimizing the difference $\left|f_{\mathrm{M}}(u)-f_{\mathrm{S}}(u)\right|$ of the observed and synthetic profiles, using the measured main beam widths $\theta_{\mathrm{b}}$ and measured main beam efficiencies $B_{\text {eff }}$, the calculated diffraction pattern $\mathcal{A}_{\mathrm{T}}$ (Sect. 5), the anticipated correlation lengths and corresponding error beams $\mathcal{F}_{\mathrm{e}}$, and also the fact that the beam structure should scale with wavelength. Figure 3 and Fig. 4 show the best-fit synthetic profiles $f_{\mathrm{S}}(u)$; the corresponding beam parameters are shown in Fig. 5.

A consistent interpretation of the measurements shown in Fig. 3 (Fig. 4) is obtained for a beam which consists of the diffracted beam $\mathcal{A}_{\mathrm{T}}$ and at least two persistent

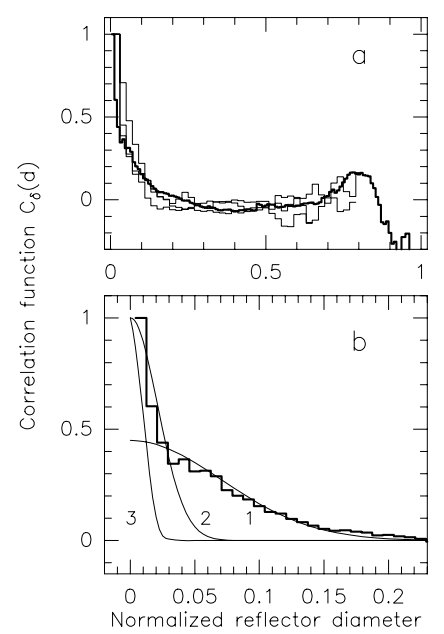

Fig. 6. Correlation functions derived from holography measurements, and Gaussian correlation length distributions calculated from Eq. (9). a) Full extent of the empirical correlation functions, for Oct. 1993, Mar. 1994, Nov. 1994: thin lines; for Sep 1996: thick line. b) Detailed view of the empirical correlation function of Sep. 1996 (histogram) and the individual Gaussian correlation functions calculated for $L_{\mathrm{p}}=0.3 \mathrm{~m}$ : curve 3; $L_{\mathrm{a}}=1.5 \mathrm{~m}$ : curve 2 ; and $L_{1}=2.5 \mathrm{~m}$ : curve 1 . The normalization is made to curve 2 (2nd EB). The lower curve 1 is due to a low night-time rms-value of the 1st EB

error beams (EB) with correlation lengths $0.3\left({ }_{-0.1}^{+0.2}\right) \mathrm{m}$ and $1.5\left({ }_{-0.1}^{+0.5}\right) \mathrm{m}$. These correlation lengths are identified with the anticipated panel surface errors $\left(L_{\mathrm{p}}\right)$ and the panel frame adjustment errors $\left(L_{\mathrm{a}}\right)$, respectively. In addition, there exists a partially transient degradation close to the main beam which may be interpreted as an error beam (1st EB) due to large-scale deformations with correlation length $3\left({ }_{-0.5}^{+1}\right) \mathrm{m}$. This transient degradation is probably due to known transient residual thermal deformations of the reflector surface and the telescope structure $^{8}$ (Greve et al. 1993, 1994b). When present, the transient thermal deformations are especially noticed during day time and sunshine (for instance as focus changes) and the comparison of measurements around New Moon and Full Moon illustrates this effect (see Fig. 4). From the correlation lengths mentioned above and the measured ratios $\mathcal{F}_{\mathrm{e}, \mathrm{i}}(0) / \mathcal{F}_{\mathrm{c}}(0)$ [Eq. (16), Eq. (11)] we derive, for the time before July 1997 , the rms-values $\sigma_{1}(1 \mathrm{st} \mathrm{EB}) \approx$ $0.03-0.06 \mathrm{~mm}$ (see the footnote of Table 1$), \sigma_{\mathrm{a}}(2 \mathrm{nd} \mathrm{EB})$ $\approx 0.07 \mathrm{~mm}$, and $\sigma_{\mathrm{p}}(3 \mathrm{rd} \mathrm{EB}) \approx 0.055 \mathrm{~mm}$. The illumination weighted rms-value $\sigma_{\mathrm{T}}=R \sqrt{\sigma_{1}^{2}+\sigma_{\mathrm{a}}^{2}+\sigma_{\mathrm{p}}^{2}} \approx 0.8 \times$ $(0.095-0.11) \approx(0.075-0.085) \mathrm{mm}$ derived in this way is consistent with the holography measurements (D. Morris,

\footnotetext{
${ }^{8}$ For instance, differential thermal dilatations of the subreflector support produce a shift of the subreflector and by this a focus offset and a slightly comatic beam. In a rigorous way, these displacements produce a deformation of the wavefront, and are not main reflector errors.
} 
Table 1. Beam parameters of the IRAM 30-m telescope (after July 1997)

\begin{tabular}{|c|c|c|c|c|}
\hline & $\begin{array}{c}\text { Main Beam }{ }^{a)} \\
\left(\mathcal{A}_{\mathrm{T}}\right)\end{array}$ & $\begin{array}{c}\text { 1st Error Beam }{ }^{b)} \\
\left(\mathcal{F}_{\mathrm{e}, 1}\right)\end{array}$ & $\begin{array}{c}\text { 2nd Error Beam }{ }^{c)} \\
\left(\mathcal{F}_{\mathrm{e}, 2}\right)\end{array}$ & $\begin{array}{c}\text { 3rd Error Beam } \\
\left(\mathcal{F}_{\mathrm{e}, 3}\right)\end{array}$ \\
\hline \multirow[t]{2}{*}{ Origin } & Diffraction & Large-scale & Panel Frame & Panel \\
\hline & Pattern & Deformations & Misalignments & Deformations \\
\hline $\begin{array}{c}\text { Correlation Length } \\
\text { Rms-value }\end{array}$ & & $\begin{array}{c}L_{1}=2.5-3.5 \mathrm{~m} \\
\sigma_{1} \lesssim 0.06 \mathrm{~mm}\end{array}$ & $\begin{array}{c}L_{\mathrm{a}}=1.5-2.0 \mathrm{~m} \\
\sigma_{\mathrm{a}}=0.055 \mathrm{~mm}\end{array}$ & $\begin{array}{c}L_{\mathrm{p}}=0.3-0.5 \mathrm{~m} \\
\sigma_{\mathrm{p}}=0.055 \mathrm{~mm}\end{array}$ \\
\hline \multicolumn{5}{|l|}{$3.4 \mathrm{~mm} / 88 \mathrm{GHz}$} \\
\hline Beam Width (FWHP) & $\theta_{\mathrm{b}}=27.5^{\prime \prime}$ & $\theta_{\mathrm{e}, 1}=300^{\prime \prime}$ & $\theta_{\mathrm{e}, 2}=410^{\prime \prime}$ & $\theta_{\mathrm{e}, 3}=2500^{\prime \prime}$ \\
\hline$\theta_{\mathrm{e}} / \theta_{\mathrm{b}}$ & & & & 90 \\
\hline Power Amplitude & $a_{\mathrm{b}}=1.00$ & $a_{\mathrm{e}, 1} \lesssim 0.0005$ & $a_{\mathrm{e}, 2}=0.0002$ & $a_{\mathrm{e}, 3}=0.000035$ \\
\hline \multicolumn{5}{|l|}{$2.0 \mathrm{~mm} / 150 \mathrm{GHz}$} \\
\hline Beam Width & $\theta_{\mathrm{b}}=16.0^{\prime \prime}$ & $\theta_{\mathrm{e}, 1}=175^{\prime \prime}$ & $\theta_{\mathrm{e}, 2}=280^{\prime \prime}$ & $\theta_{\mathrm{e}, 3}=1500^{\prime \prime}$ \\
\hline$\theta_{\mathrm{e}} / \theta_{\mathrm{b}}$ & & & & 95 \\
\hline Power Amplitude & $a_{\mathrm{b}}=1.00$ & $a_{\mathrm{e}, 1} \lesssim 0.0015$ & $a_{\mathrm{e}, 2}=0.00055$ & $a_{\mathrm{e}, 3}=0.000055$ \\
\hline \multicolumn{5}{|l|}{$1.3 \mathrm{~mm} / 230 \mathrm{GHz}$} \\
\hline Beam Width & $\theta_{\mathrm{b}}=10.5^{\prime \prime}$ & $\theta_{\mathrm{e}, 1}=125^{\prime \prime}$ & $\theta_{\mathrm{e}, 2}=180^{\prime \prime}$ & $\theta_{\mathrm{e}, 3}=950^{\prime \prime}$ \\
\hline$\theta_{\mathrm{e}} / \theta_{\mathrm{b}}$ & & 12 & & 90 \\
\hline Power Amplitude & $a_{\mathrm{b}}=0.975$ & $a_{\mathrm{e}, 1} \lesssim 0.005$ & $a_{\mathrm{e}, 2}=0.001$ & $a_{\mathrm{e}, 3}=0.00009$ \\
\hline \multicolumn{5}{|l|}{$0.86 \mathrm{~mm} / 350 \mathrm{GHz}$} \\
\hline Beam Width & $\theta_{\mathrm{b}}=8.5^{\prime \prime}$ & $\theta_{\mathrm{e}, 1}=85^{\prime \prime}$ & $\theta_{\mathrm{e}, 2}=160^{\prime \prime}$ & $\theta_{\mathrm{e}, 3}=580^{\prime \prime}$ \\
\hline$\theta_{\mathrm{e}} / \theta_{\mathrm{b}}$ & & 10 & 19 & 70 \\
\hline Power Amplitude & $a_{\mathrm{b}}=0.975$ & $a_{\mathrm{e}, 1} \lesssim 0.008$ & $a_{\mathrm{e}, 2}=0.002$ & $a_{\mathrm{e}, 3}=0.00025$ \\
\hline
\end{tabular}

a) The diffraction pattern $\mathcal{A}_{\mathrm{T}}$ is discussed in Sect. 5 .

b) The 1st EB is a partially transient phenomenon which may show, in particular, a day-night time effect due to residual transient thermal deformations.

The lower limits of $\sigma_{1}$ and $a_{\mathrm{e}, 1}$, under best conditions, are $\sim 1 / 2$ of the listed values.

c) The values valid before July 1997 are larger by $\sim 40 \%$ (see Sect. 4); the values $a_{\mathrm{e}, 1}$ and $a_{\mathrm{e}, 3}$ remain unchanged.

priv. comm.) and the efficiency measurements of the planets (Kramer 1997), as it should be the case.

Figure 5 shows the widths $\theta_{\mathrm{e}}$ and amplitudes $a_{\mathrm{e}}$ of the error beams as derived from the measurements and calculated from Eqs. $(11,12)$ for $\epsilon_{0}=0.62$ (Greve et al. 1994c) and the correlation lengths $L_{i}$ and rms-values $\sigma_{i}$ determined above. This figure confirms the wavelengthscaling of the error beams. When present, the wavelengthscaling of the 1st error beam indicates that the associated large-scale deformations behave like random deformations with correlation length $L_{1}=2.5-3.5 \mathrm{~m}$, of which there are approximately $\left(D / L_{1}\right)^{2} \approx 50$ elements covering the aperture. This large number of deformation patches allows the application of statistical calculations (Greve 1980).

\subsection{The empirical correlation function}

The wavefront errors $\left(\delta_{\varphi}\right)$ derived from holography measurements corrected for defocus and coma (see footnote 8 ) are interpreted to be due to surface errors $(\delta)$ of the main reflector. This surface error distribution $[\delta]$ can be used to derive the surface error correlation function $\left[C_{\delta}\right]$ and from this, in an independent way, the correlation length(s) $[L]$. Holography measurements at $7 \mathrm{~mm}(43 \mathrm{GHz})$ wavelength are regularly made at $43^{\circ}$ elevation using the geostationary satellite ITALSAT (Morris et al. 1996, 1997). At this elevation the reflector surface is optimized and free of homology deformations (Greve et al. 1998). We derived the correlation function $C_{\delta}$ (Eq. (14)), shown in Fig. 6 , from $32 \times 32$ pixel holography measurements made Oct. 1993, Mar. 1994, Nov. 1994, and from a $128 \times 128$ pixel holography measurement of $0.24 \mathrm{~m}(=D / 128)$ spatial resolution made Sep. 1996 (Morris et al. 1996). When compared with Gaussian correlation length distributions $\exp \left[-(d / L)^{2}\right]$ (Eq. (9)), the empirical correlation functions show clearly the influence of the panel frame misalignment (2 in Fig. 6b), with correlation length $L_{\mathrm{a}}=1.5-2.0 \mathrm{~m}$, and of the large-scale deformations ( 1 in Fig. $6 \mathrm{~b}$ ), with correlation length $L_{1}=2.5-3.5 \mathrm{~m}$. The resolution of the $128 \times$ 128 pixel holography map with one or two measurements per correlation cell $L_{\mathrm{p}}=0.3-0.5 \mathrm{~m}$ is too low to clearly indicate the Gaussian component of the panel deformations (3 in Fig. 6b). However, the panel surface errors of correlation length $L_{\mathrm{p}}$ are clearly illustrated in Fig. 1.

\subsection{The modified Ruze relation}

The aperture efficiency $\epsilon_{\mathrm{ap}}$ of a reflector with several independent error distributions is (Ruze 1966; Shifrin 1971; Baars 1973)

$\epsilon_{\mathrm{ap}}=\epsilon(R)_{\mathrm{ap}}+\epsilon(L)_{\mathrm{ap}}$

$=\epsilon_{0} \exp \left[-\sum_{i}\left(\sigma_{\varphi, i}\right)^{2}\right]+\sum_{i}\left(1-\exp \left[-\left(\sigma_{\varphi, i}\right)^{2}\right]\right)\left(L_{i} / D\right)^{2}(20)$ 
where

$\epsilon(R)_{\text {ap }}=\epsilon_{0} \exp \left[-\sum_{i}\left(\sigma_{\varphi, i}\right)^{2}\right]=\epsilon_{0} \exp \left[-\left(\sigma_{\varphi}\right)^{2}\right]$

is the standard Ruze relation and $\epsilon(L)_{\text {ap }}$ a correction taking into account the scale of the surface errors. It is evident from Eq. (20) that in particular large-scale deformations (large $L$ ) contribute to $\epsilon(L)_{\text {ap }}$, increasing the efficiency $\epsilon(R)_{\text {ap }}$. When using in Eqs. $(20,21)$ the measured values $\left(\sigma_{i}, L_{i}\right)$ given in Table 1 , and $\epsilon_{0}=$ 0.62 , we find that in the wavelength region from $0.8 \mathrm{~mm}$ to $3 \mathrm{~mm}$ the quantity $\epsilon(L)_{\text {ap }}$ does not exceed $1-2 \%$, which is below the accuracy of the measurements. The difference between the Ruze relation $(L=0)$ and the complete expression Eq. (20) is so small so that the Ruze relation can be used for evaluation of the $30-\mathrm{m}$ reflector.

\section{The current surface accuracy}

We have presented in detail the data taken before July 1997 since this complete set of multi-wavelength Moon scans (Fig. 3) and corresponding beam parameters (Fig. 5) illustrate that a consistent theory exists to describe the beam degradation from several surface error distributions. In this theory it is possible to anticipate the structure of the beam pattern and the number of beam components from details of the reflector surface construction. Although it may be possible to approximate the measurements by some other analytic function than Eqs. $(10,16)$, the decomposition used here is consistent with proven concepts of the antenna tolerance theory and with the surface structure of the $30-\mathrm{m}$ reflector. We believe that the use of a priori knowledge of the beam structure allows the derivation of meaningful parameters of the error beams, in particular of very extended low level error beams which are difficult to measure with high precision. We believe also that the predictability of the beam structure from details of the reflector construction allows a meaningful estimate of the influence of surface improvements, as done in the following.

It is evident from the $30-\mathrm{m}$ reflector construction that the correlation lengths $L_{\mathrm{p}}$ (panels, 3rd EB) and $L_{\mathrm{a}}$ (panel frames, 2nd EB) are fixed quantities, hence also the widths $\theta_{\mathrm{e}, \mathrm{p}}$ and $\theta_{\mathrm{e}, \mathrm{a}}$ of the corresponding error beams. Since only panel frames can be adjusted, only the associated rms-value $\sigma_{\mathrm{a}}$ may change, leading to the improved value $\sigma_{\mathrm{a}}^{*}$. The associated change of the power amplitude $a_{\mathrm{e}, a}$ (Eq. (11), Fig. 5) of the corresponding 2nd error beam is $a_{\mathrm{e}, \mathrm{a}}^{*} / a_{\mathrm{e}, \mathrm{a}}=\left[1-\exp \left(-\sigma_{\varphi, \mathrm{a}}^{* 2}\right)\right] /\left[1-\exp \left(-\sigma_{\varphi, \mathrm{a}}^{2}\right)\right]$.

Approximately $80 \%$ of the power removed by the surface adjustment from the error beam appears as an increase in power of the diffracted main beam, the remaining $\sim 20 \%$ appear in the sidelobe pattern (see Born \& Wolf 1980).

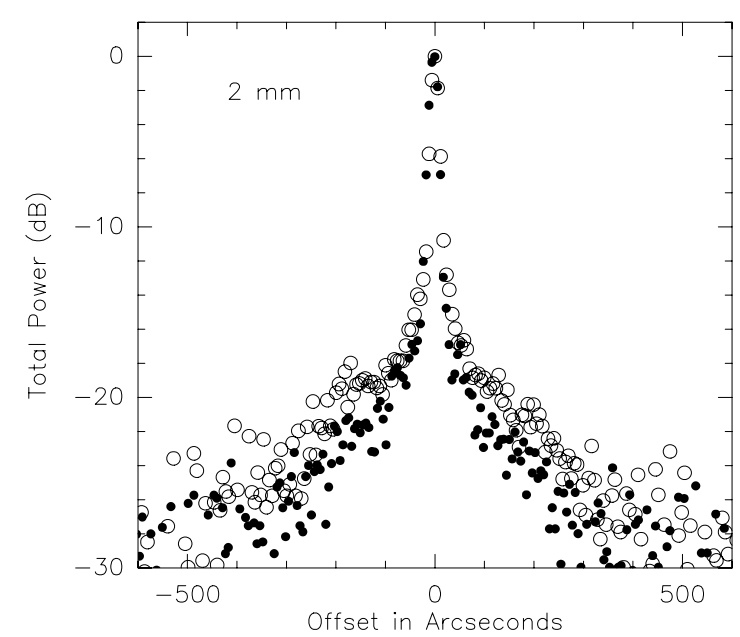

Fig. 7. Composite profiles $f_{\mathrm{M}}(u)$ which illustrate the improvement of the reflector surface accuracy; measurements before July 1997 (24 Dec. 1994): open circles, after July 1997 (19 Nov. 1997): solid dots

A holography measurement (Morris et al. 1997) has shown that the panel frame adjustment of July 1997 has improved $^{9}$ the illumination weighted reflector surface accuracy from $\sigma_{\mathrm{T}}=0.075-0.085 \mathrm{~mm}$ to $\sigma_{\mathrm{T}}^{*}=0.065-$ $0.075 \mathrm{~mm}$. This general improvement is due to a reduction of the panel frame rms-value from $\sigma_{\mathrm{a}, \mathrm{T}} \approx 0.07 \mathrm{~mm}$ to $\sigma_{\mathrm{a}, \mathrm{T}}^{*} \approx 0.055 \mathrm{~mm}$. Using Eq. (22), the associated reduction of the power amplitude of the 2 nd error beam is $a_{\mathrm{e}, \mathrm{a}}^{*}$ $\equiv a_{\mathrm{e}, 2}^{*} \approx 0.6 a_{\mathrm{e}, \mathrm{a}}$.

In addition to the holography measurements, we confirmed the improvement of the reflector surface precision from a measurement of the beam pattern and of the aperture efficiency at $0.86 \mathrm{~mm}(350 \mathrm{GHz})$ :

- at the lunar age of 21.3 days ( Last Quarter, 19 Nov. 1997) we obtained with the improved reflector a scan across the Moon at $2 \mathrm{~mm}$ wavelength, and constructed from this the composite profile shown in Fig. 7. From an earlier observation we constructed a composite profile for the lunar age of 19.6 days (24 Dec. 1994), also shown in Fig. 7. The observations are sufficiently close in phase to allow a comparison of both profiles (see the Appendix). The improvement of the reflector surface is evident in Fig. 7 as a reduction of the error beam.

- we derived in 1994 and 1998 the aperture efficiency at $0.86 \mathrm{~mm}(350 \mathrm{GHz})$ from measurements of the planets, using the same SIS receiver (see Table 2). The measured increase of the aperture efficiency from $\sim 12 \%$ to $\sim 16 \%$ (Table 2) agrees with the improvement of the reflector surface.

9 The rms-values before July 1997 are (in $\mathrm{mm}$ ): $\sigma_{1} \approx 0.03-$ $0.06, \sigma_{\mathrm{a}} \approx 0.07, \sigma_{\mathrm{p}} \approx 0.055$ so that $\sigma_{\mathrm{T}}=R \sigma=0.075-0.085$; after July 1997 they are: $\sigma_{1} \approx 0.03-0.06, \sigma_{\mathrm{a}} \approx \sigma_{\mathrm{p}} \approx 0.055$ so that $\sigma_{\mathrm{T}}=R \sigma=0.065-0.075$ (see Table 1 ). 

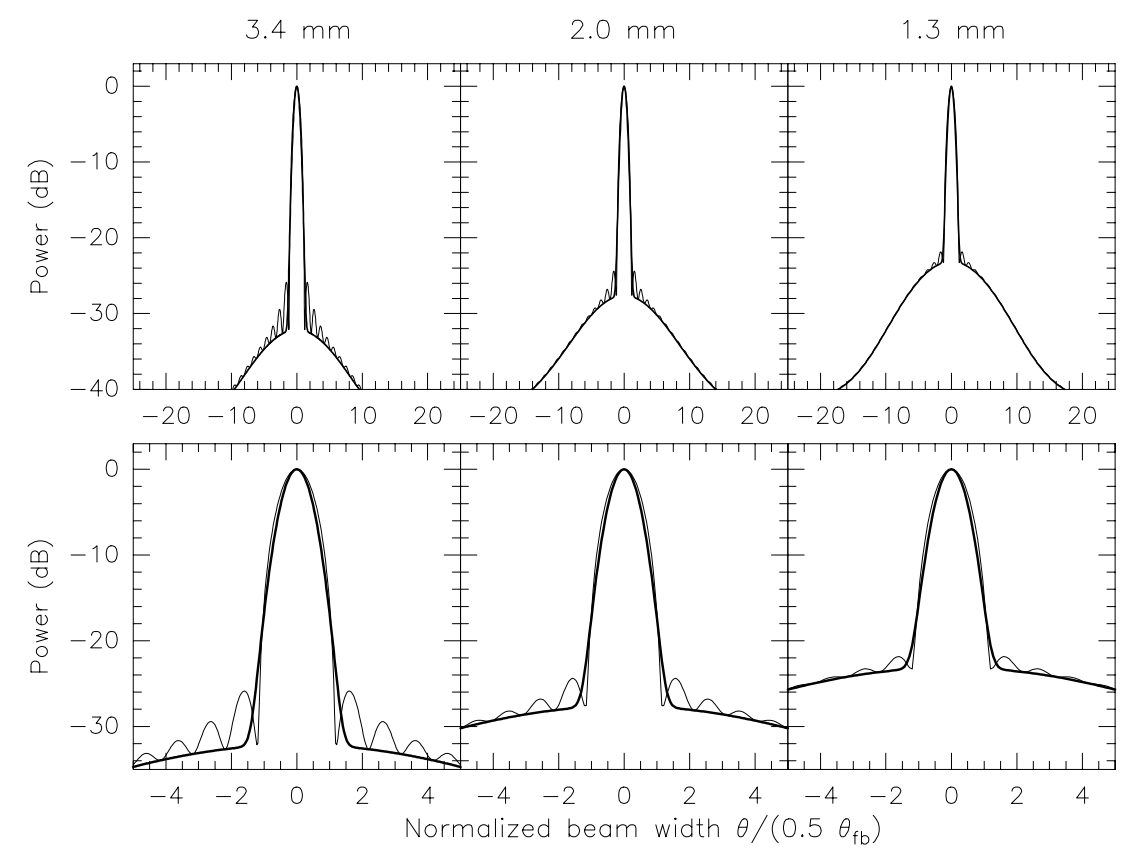

Fig. 8. IRAM 30-m telescope current beam pattern calculated from Eq. (16) and the parameters of Table 1. The heavy lines show the case of a Gaussian approximation of the main beam (Eq. (23)). The accuracy of the profiles is $\sim \pm 1 \mathrm{~dB}$ beyond the main beam area. The beam patterns are shown on logarithmic scale $(\mathrm{dB})$. For each wavelength the profile is normalized to $1 / 2$ width of the full beam $\theta_{\mathrm{fb}}$ (see Table 2)

Using these data, we proceeded in the following way to arrive at representative parameters of the telescope performance for the time after the July 1997 surface adjustment:

(1) we use the values $\theta_{\mathrm{e}}$ and $a_{\mathrm{e}}$ derived from the multiwavelength set of Moon scans (Fig. 5), but update the values $a_{\mathrm{e}, 2}=a_{\mathrm{e}, \mathrm{a}}$ (panel frames, 2nd EB) by application of Eq. (22) as explained above. In Eq. (22) we use the values $\sigma_{\mathrm{a}, \mathrm{T}}=0.070 \mathrm{~mm}$ and $\sigma_{\mathrm{a}, \mathrm{T}}^{*}=0.055 \mathrm{~mm}$ based on the earlier and recent holography measurements (see footnote 9).

(2) we use the holography measurement of the reflector surface precision $\left(\sigma_{\mathrm{T}}^{*}\right)$ and of the $350 \mathrm{GHz}$ aperture efficiency to update the earlier efficiency data compiled by Kramer (1997) from a large set of observations, as not yet available for the improved reflector.

The current beam parameters are shown in Fig. 5 and are given in Table 1; the current efficiencies are given in Table 2.

\section{The current beam pattern}

Figure 8 shows the current beam pattern at $3.4 \mathrm{~mm}$, $2.0 \mathrm{~mm}$, and $1.3 \mathrm{~mm}$ wavelength calculated from $\mathrm{Eq}$. (16) and the values of Table 1 . The values of Table 1, the curves of Fig. 5, and Eqs. $(11,12)$ can be used to derive the parameters of the beam pattern for other wavelengths than the reported measurements.

The calculation of the beam pattern requires a knowledge of the diffraction pattern $\mathcal{A}_{\mathrm{T}}$. Without entering into lengthy calculations (see Minnett \& Thomas
1968; Goldsmith 1987), the diffraction pattern of the 30-m telescope is obtained with sufficient accuracy, when compared to measurements, from the approximation $\mathcal{A}_{\mathrm{T}}(u)=\alpha(u) \mathcal{A}(u)$, with $\mathcal{A}(u)=\left[J_{1}(u) / u\right]^{2}$ calculated from the expressions given by Abramowitz \& Stegun (1972) and the reduction factor $\alpha(u)=0.12,0.22,0.27$ for the 1st, 2nd, and 3rd sidelobe. In this calculation the beam width (FWHP) of the Airy pattern $\mathcal{A}(u)$ is $\theta_{\mathrm{b}}$ (Table 1 ) at $u=1.62$. As evident from Fig. 8 , at the level of the 3rd sidelobe the diffraction pattern and the error pattern have similar intensities. The sidelobe structure and the error beam seen in Fig. 8 are not observed in regular pointing scans made with the $30-\mathrm{m}$ telescope because the sensitivity of the procedure is only $-10 \mathrm{~dB}$ to $-15 \mathrm{~dB}$. The sidelobe structure is also not seen in the composite Moon scans (Figs. 3, 4) since this detail is lost in the convolution (Eq. (18)).

In many applications the diffracted beam $\mathcal{A}_{\mathrm{T}}$ is approximated by a Gaussian main beam without sidelobes

$\mathcal{A}_{\mathrm{T}} \approx \mathcal{G}_{\mathrm{T}}=\exp \left[-(\theta / \Theta)^{2}\right]$

with $\Theta=\theta_{\mathrm{b}} /(2 \sqrt{\ln 2})=0.60 \theta_{\mathrm{b}}$. Figure 8 shows also the approximated Gaussian main beams.

\section{The current efficiencies}

The current efficiencies given in Table 2 are primarily based on the measured illumination weighted rms-value $\sigma_{\mathrm{T}}^{*}$. The aperture efficiency $\epsilon_{\mathrm{ap}}$ and the beam efficiency $B_{\text {eff }}$ are related by $B_{\text {eff }}=0.8899\left[\theta_{\mathrm{b}} /(\lambda / D)\right]^{2} \epsilon_{\mathrm{ap}} \approx 1.20 \epsilon_{\mathrm{ap}}$ 


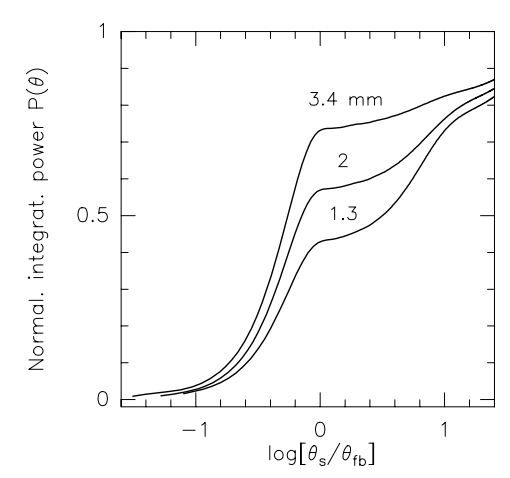

Fig. 9. Relative power $P(\Omega)$ (Eq. (24)) received in the solid angle $\Omega$ of opening $\theta_{\mathrm{s}}$ given in fractions of the full beam width $\theta_{\mathrm{fb}}$ (Table 2). $P(\Omega)$ at $\theta_{\mathrm{s}} / \theta_{\mathrm{fb}}=1$ is the beam efficiency $B_{\mathrm{eff}}$; the normalization of the curves is made to these values given in Table 2. The values are shown for $\theta_{\mathrm{s}} \lesssim 1000-1400^{\prime \prime}$, i.e. the extent of the profile measurements where also $F_{\text {eff }} \approx \epsilon_{\mathrm{M}}$ (Table 2). The remaining energy for larger angles $\theta_{\mathrm{s}}$ is mainly in the backward beam and is of the order $1-F_{\text {eff }}$

(Downes 1989), when assuming a Gaussian main beam and using relation (1).

The relative power $P(\Omega)$ received in the on-axis solid angle $\Omega$ of opening $\theta_{\mathrm{s}}$ is

$P(\Omega)=\sum_{i=0,3} \int_{0}^{\Omega} \mathcal{F}_{i}\left(\Omega^{\prime}\right) \mathrm{d} \Omega^{\prime} / \sum_{i=0,3} \int_{0}^{4 \pi} \mathcal{F}_{i}\left(\Omega^{\prime}\right) \mathrm{d} \Omega^{\prime}$

In Eq. (24), $\mathcal{F}_{0}$ is the diffracted beam $\mathcal{A}_{\mathrm{T}}$ and $\mathcal{F}_{i}(i=$ $1,2,3)$ are the error beams. When using the diffracted Gaussian main beam $\mathcal{G}_{\mathrm{T}}$ we obtain for $P(\Omega)=P\left(\theta_{\mathrm{s}} / 2\right)$ the relation

$P(\Omega)=\sum_{i=0,3} a_{i} \Theta_{i}^{2}\left[1-\exp \left(-\left[\left(\theta_{\mathrm{s}} / 2\right) / \Theta_{i}\right]^{2}\right)\right] / \sum a_{i} \Theta_{i}^{2}$.

The function $P(\Omega)$, based on the parameters of Table 1 and Table 2, is shown in Fig. 9 for $\lambda=3 \mathrm{~mm}, 2 \mathrm{~mm}$, and $1.3 \mathrm{~mm}$. In particular, the beam efficiency is $B_{\text {eff }}=P\left(\Omega_{\mathrm{fb}}\right)$ with $\Omega_{\mathrm{fb}}$ the solid angle of the full beam of diameter $\theta_{\mathrm{fb}}$ as given in Table 2 .

For observations of extended sources it is important to know the fraction of the power contained in the diffracted beam and the error beams. The integrated relative power $P_{i}$ of the beam component $[i]$ is

$P_{i}=\int_{0}^{4 \pi} \mathcal{F}_{i}(\Omega) \mathrm{d} \Omega / \sum_{i=0,3} \int_{0}^{4 \pi} \mathcal{F}_{i}(\Omega) \mathrm{d} \Omega$

or when using the diffracted Gaussian main beam $\mathcal{G}_{\mathrm{T}}$ we obtain

$P_{i}=a_{i} \Theta_{i}^{2} / \sum a_{i} \Theta_{i}^{2}$.

Table 2 gives the values $P_{i}(i=1,2,3)$ calculated from Eq. (27) and the parameters of Table 1. The total power contained in the error beams is $P_{\mathrm{e}}=\sum P_{i}$.

From the calibrated scans taken at New Moon (Fig. 10a) and the temperature of the New Moon published by Fedoseev \& Chernyshev (1998) we derived the Moon efficiency $\epsilon_{\mathrm{M}}$ of which the values are given in
Table 2. The similarity of the values of the measured forward efficiency $\left(F_{\text {eff }}\right.$, Table 2$)$ and of the Moon efficiency says that the forward beam has approximately the size of the Moon's disk. The similarity of the values also says that the calculated beam pattern should not be used beyond the off-axis distance of $\sim 700-900^{\prime \prime}$, a fact we respected in Fig. 8 and Fig. 9. It is evident that caution is required when using Eq. (24)-Eq. (27) which imply integrations over the full extent of the beam. The accuracy of the values $P(\Omega)$ and $P_{i}$ (Fig. 9, Table 2) is a few percent.

\section{Summary}

1) For the analysis of the $30-\mathrm{m}$ telescope beam we have used the established method of total power scans across the limb of the Moon. We did not apply a rigorous deconvolution but instead obtained the beam parameters from a more direct best-fit comparison between observed and synthetic profiles. The method works well for measurements around New Moon and Full Moon.

2) The antenna tolerance theory of several independent large-scale and small-scale wavefront (reflector surface) deformations provides a consistent description of the 30-m telescope beam. This theory makes use of the concept of individual correlation lengths $\left(L_{i}\right)$ and individual rms-values $\left(\sigma_{i}\right)$ of the independent error distributions $\left[\delta_{i}\right]$. An estimate of the correlation lengths can be obtained from details of the reflector surface construction, in particular for a design of (mini-)panels and panel frames.

3) We find from the analysis of the 30-m reflector that the Gaussian correlation length distribution is a good representation of the actual situation. The observed wavelength-scaling of the error beams gives confidence in the correctness of the theoretical predictions and the empirical values.

4) The effective rms-value of the wavefront (reflector surface) is the root-square-sum of the individual rmsvalues $\sigma_{i}$. As expected, this value agrees for the $30-\mathrm{m}$ reflector with the effective rms-value obtained from holography data and efficiency measurements. Compared with the errors of efficiency measurements, the standard Ruze relation (for $L_{i}=0$ ) can be applied on the 30-m telescope without loss of precision.

5) Any improvement of a paneled reflector surface with several error distributions should act, if possible, on all error components which, however, dependent of the reflector construction, may not always be possible. It is important to consider also the correction of large-scale wavefront deformations (for instance by using adaptive optics, Greve et al. 1996) which deform the central area of the beam and which may contain a significant amount of the total power.

6) Any further improvement of the $30-\mathrm{m}$ reflector surface can, at the present state, only be made by further improvement of the panel frame adjustment. In this case, 
Table 2. Efficiency parameters of the IRAM 30-m telescope (after July 1997)

\begin{tabular}{|c|c|c|c|c|c|c|c|c|c|c|}
\hline $\begin{array}{l}\text { Wavel./Freq. } \\
{[\mathrm{mm}] /[\mathrm{GHz}]}\end{array}$ & $\begin{array}{l}\theta_{\mathrm{b}} \\
{\left[{ }^{\prime \prime}\right]}\end{array}$ & $\begin{array}{l}\theta_{\mathrm{fb}} \\
{\left[{ }^{\prime \prime}\right]}\end{array}$ & $\begin{array}{l}\epsilon_{\mathrm{ap}} \\
{[\%]}\end{array}$ & $\begin{array}{l}B_{\text {eff }} \\
{[\%]}\end{array}$ & $\begin{array}{l}F_{\text {eff }} \\
{[\%]}\end{array}$ & $\begin{array}{c}\epsilon_{\mathrm{M}} \\
{[\%]}\end{array}$ & $\begin{array}{c}S / T_{\mathrm{A}}^{*} \\
{[\mathrm{Jy} / \mathrm{K}]}\end{array}$ & $\begin{array}{r}P_{1}\left(\theta_{\mathrm{e}, 1}\right) \\
{[\%]\left(^{\prime \prime}\right)}\end{array}$ & $\begin{array}{r}P_{2}\left(\theta_{\mathrm{e}, 2}\right) \\
{[\%]\left({ }^{\prime \prime}\right)}\end{array}$ & $\begin{array}{r}P_{3}\left(\theta_{\mathrm{e}, 3}\right) \\
{[\%]\left(^{\prime \prime}\right)}\end{array}$ \\
\hline $3.4 / 88$ & 27.5 & $\sim 64$ & $61 \pm 3$ & $73 \pm 3$ & $2 \pm 2$ & $94 \pm 4$ & $5.9 \pm 0.3$ & $2-4(300)$ & $3(410)$ & $20(2500)$ \\
\hline $2.0 / 150$ & 16.0 & $\sim 38$ & $45 \pm$ & $54 \pm$ & $90 \pm$ & $92=$ & $7.8 \pm 0.5$ & $5-10(175)$ & $8(280)$ & $25(1500)$ \\
\hline $1.3 / 230$ & 10.5 & $\sim 25$ & $35 \pm 3$ & $42 \pm 3$ & $86 \pm 2$ & & $9.7 \pm 0.9$ & $10-20(125)$ & $12(180)$ & $26(950)$ \\
\hline$[0.86 / 350$ & 8.5 & $\sim 20$ & $16 \pm 4$ & $19 \pm 4$ & $75 \pm 3$ & & $22 \pm 3$ & $15-25(85)$ & $20(160)$ & $\left.30(580)]^{*}\right)$ \\
\hline
\end{tabular}

Update from the values compiled by Kramer (1997).

The entries of the Table are:

$\theta_{\mathrm{b}}$ : beam width (FWHP) (measured); $\theta_{\mathrm{fb}}$ : full width (to first minimum), $\theta_{\mathrm{fb}} \approx 2.4 \theta_{\mathrm{b}}$ (calculated);

$\epsilon_{\mathrm{ap}}$ : aperture efficiency (measured \& calculated from $\sigma_{\mathrm{T}}^{*}$ ); $B_{\mathrm{eff}}$ : main beam efficiency, $B_{\mathrm{eff}} \approx 1.20 \epsilon_{\mathrm{ap}}$;

$F_{\text {eff }}$ : forward efficiency (from sky dips), $\epsilon_{\mathrm{M}}$ : Moon efficiency (measured);

$S / T_{\mathrm{A}}^{*}=(2 \mathrm{k} / A) F_{\mathrm{eff}} / \epsilon_{\mathrm{ap}}=3.906 F_{\mathrm{eff}} / \epsilon_{\mathrm{ap}}$ : antenna gain (calculated \& measured).

$P_{1}-P_{3}$ : relative power of the error beams (calculated). The accuracy of the values is $\sim \pm 5 \%$. The entries of $P_{1}$

illustrate the partially transient nature of this error beam. In brackets are given the widths (FWHP) of the

corresponding error beams.

* Not frequently used frequency and somewhat poorly known telescope performance.

The values valid before July 1997 are published by Kramer (1997) and are found in the 30-m Telescope Manual (Wild).

the parameters of the improved beam are obtained in the way as outlined in Sect. 4, supported by Moon scan measurements.

\section{Appendix A: Limb scans at various phases of the Moon}

For the analysis of the beam structure we have used scans across the Moon taken around New Moon and Full Moon. In order to derive in an empirical way the parameters of the beam pattern, we have constructed from the observed total power scans $P_{\mathrm{M}}(u)$ the differentiated, composite profiles $f_{\mathrm{M}}(u)$ which we compared with synthetic profiles $f_{\mathrm{S}}(u)$ (Eq. (19)), obtained from synthetic total power scans $P_{\mathrm{S}}(u)$ (Eq. (18)). There are two reasons for selecting observations around New Moon (day time) and Full Moon (night time): (a) at these phases the Moon's brightness distribution is symmetric with respect to its center and easily cast into an analytic form (Eq. (17)) for calculation of the synthetic profiles $P_{\mathrm{S}}(u)$ and $f_{\mathrm{S}}(u)$; (b) the observed total power scans $P_{\mathrm{M}}(u)$ and the differentiated scans $f_{\mathrm{M}}(u)$ are identical for any scan direction through the center of the Moon so that opposite sides of the limb can be used to construct a composite profile $f_{\mathrm{M}}(u)$. Both segments of a composite profile are normalized at their center.

We illustrate these remarks with observations. Figure 10a shows $2 \mathrm{~mm}$ wavelength total power scans at different phases of the Moon. In these observations, one scan passes through the subsolar point and the center ( $\sim$ EW direction at culmination); the other scan is made in orthogonal direction. The figure shows the symmetric brightness distribution around New Moon (age $\approx 0$ days) and Full Moon (age $\approx 15$ days). As shown in Fig. 10b and Fig. 10c, at these phases the profiles $f_{\mathrm{M}}$ (East limb) and $f_{\mathrm{M}}$ (West limb) are comparable so that the measurements at opposite limbs can be used to construct a composite profile $f_{\mathrm{M}}(u)$, as done above (see Figs. 3,4$)$. We find empirically that the difference of the profile sections $f_{\mathrm{M}}$ (East) and $f_{\mathrm{M}}$ (West) is below $\sim 1 \mathrm{~dB}$, and hence below the accuracy of the measurements, for observations within $\sim \pm 1$ day of New Moon and Full Moon. Also, at these phases of the Moon the unbalance of the profiles because of temperature gradients across the Moon and limb darkening is smaller than the accuracy of the measurements. From observed and synthesized scans we find that the composite profiles are identical for measurements at New Moon and Full Moon (see Fig. 10c). This allows a comparison of profiles taken at New Moon and Full Moon, as done in Fig. 4. We find also that profiles taken in $u$-direction or $v$-direction are similar within $\sim \pm 1 \mathrm{~dB}$ when taken within $\sim \pm 1$ day of a certain phase, as used in Fig. 7 .

The brightness distribution at other phases of the Moon than New Moon and Full Moon shows a more or less steep gradient and a more or less pronounced crescent (Fig. 10a). The disadvantage of using for the analysis other phases than New Moon and Full Moon lies in the fact that the available models (Krotikov \& Troitskii 1964; Linsky 1966, 1973; Mangum 1993) for calculation of the Moon's millimeter wavelength brightness distribution $T_{0} \Pi(u, v)$ (Eqs. $\left.(17,18)\right)$ are not sufficiently precise, and mathematically cumbersome, to produce reliable synthetic profiles $P_{\mathrm{S}}(u)$ and $f_{\mathrm{S}}(u)$ for comparison with observations.

The $\mathrm{mm}$-wavelength radiation from the edge of the Moon is polarized in radial direction by $\sim 1 \%$ at $1.3 \mathrm{~mm}$ (Barvainis et al. 1988). This is a small and systematic effect and need not be considered in the profile analysis, even when using polarized feeds.

Acknowledgements. The holography data were kindly made available by D. Morris. The holography data of 1996 and 1997 were obtained in a larger IRAM campaign including the 


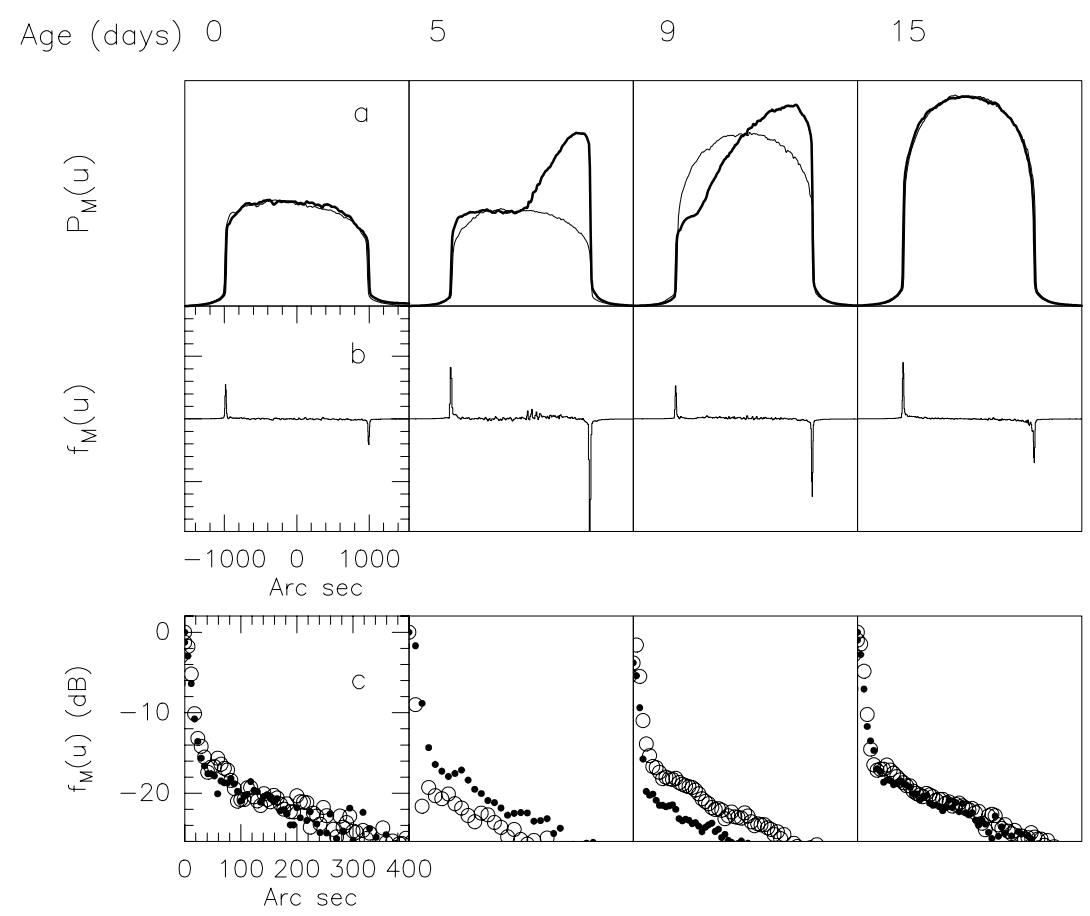

Fig. 10. a) Observed (before July 1997) total power scans at $2 \mathrm{~mm}$ wavelength; West is to the right, East to the left. The age of the Moon is indicated as days after New Moon (scans of the last half of the lunar cycle are similar, with the EW direction inverted). The profiles show scans $P_{\mathrm{M}}(u)$ through the subsolar point (i.e. $\sim$ EW direction at culmination) and the center of the Moon (thick line), and scans in orthogonal direction (thin line). Only scans at New Moon and Full Moon are symmetric. b) Moon scans differentiated along $\sim$ EW direction, i.e. $f_{\mathrm{M}}(u)=\mathrm{d} P_{\mathrm{M}}(u) / \mathrm{d} u$. c) Sections of the composite profile $f_{\mathrm{M}}$ with $f_{\mathrm{M}}$ (West): solid dots, $f_{\mathrm{M}}$ (East): open circles (folded around the beam axis)

collaboration of D. Morris, J. Lamb, B. Lazareff, M. Carter, F. Mattiocco, and the staff of IRAM-Granada. D. Teyssier of IRAM-Granada made the new measurements used in Fig. 7; A. Karpov (IRAM) provided and operated the $350 \mathrm{GHz}$ receiver. The data of Fig. 1 are taken from the ARGE-Krupp-MAN documentation of the telescope. We appreciated the discussions with D. Morris and B. Lazareff, and the pointed comments of D. Downes (IRAM) and the referee, R.E. Hills.

\section{References}

Abramowitz M., Stegun I.A., 1972, Handbook of Mathematical Functions. Dover Publ. Inc., New York

Baars J.W.M., 1973, IEEE Trans. Ant. Propag. AP-21, 461

Baars J.W.M., Hooghoudt B.G., Mezger P.G., de Jonge M., 1987, A\&A 175, 319

Baars J.W.M., Greve A., Hein H., et al., 1994, Proc. IEEE 82, 687

Barvainis R., Clemens D.P., Leach R., 1988, PASP 95, 510

Born M., Wolf E., 1980, Principles of Optics. Pergamon Press, Oxford, UK

Christiansen W.N., Högbom J.A., 1985, Radiotelescopes. Cambridge University Press, Cambridge, UK

Cortes-Medellin G., Goldsmith P.F., 1994, IEEE Trans. Ant. Propag. AP-42, 176

Downes D., 1989, Radio Astronomy Techniques, in Evolution of Galaxies, Astronomical Observations, Appenzeller I., Habing H.J., Lena P. (eds.). Springer Verlag, Heidelberg
Fedoseev L.I., Chernyshev V.I., 1998, Astronomy Reports 42, 105

Garcia-Burillo S., Guelin M., Cernicharo J., 1993, A\&A 274, 144

Goldsmith P.F., 1987, Int. J. Infrared \& Millimeter Waves 8, 771

Greve A., 1980, Appl. Opt. 19, 2948

Greve A., Hooghoudt B.G., 1981, A\&A 93, 76

Greve A., Dan M., Penalver J., 1993, IEEE Trans. Ant. Propag. $\mathrm{AP}-40,1375$

Greve A., Morris D., Johansson L.E.B., et al., 1994a, IEE Proc. Microw. Ant. Propag. 141, 23

Greve A., LeFloch B., Morris D., et al., 1994b, IEEE Trans. Ant. Propag. AP-42, 1345

Greve A., Steppe H., Graham D.A., et al., 1994c, A\&A 286, 654

Greve A., Panis J.-F., Thum C., 1996a, A\&AS 115, 379

Greve A., Baars J.W.M., Penalver J., et al., 1996b, Radio Sci. 31,1053

Greve A., Neri R., Sievers A., 1998, A\&AS (in print)

Harris J.W., Catalan G., Atad-Ettedgui E., et al., 1997, Appl. Opt. 36, 4571

Hills R.E., Richer J., 1992, The JCMT Newsletter, Aug. 24

Horne K., Hurford G.J., Zirin H., 1981, ApJ 244, 340

Kaufmann P., Abraham Z., Scalise E., et al., 1987, IEEE Trans. Ant. Propag. AP-35, 996

Kramer C., 1997, Calibration of spectral line data at the IRAM $30 \mathrm{~m}$ Telescope, IRAM Internal Report

Kraus J.D., 1986, Radioastronomy. Cygnus-Quasar Books, Powell, Ohio, U.S.A. 
Krotikov V.D., Troitskii V.S., 1964, Sov. Phys.-Ups. 6, 841

Lindsey C.A., Roellig T.L., 1991, ApJ 375, 414

Linsky J.L., 1966, Icarus 5, 606

Linsky J.L., 1973, ApJS 25, 163

Love A.W., 1978, Reflector Antennas. IEEE Press, New York

Mangum J.G., 1993, PASP 105, 117

Marechal A., 1947, Rev. Opt. 26, 257

Mauersberger R., Guelin M., Martin-Pintado J., et al., 1989, A\&AS 79,217

Minnet H.C., Thomas B.M., 1968, Proc. IEE 115, 1419

Morris D., Baars J.W.M., Hein H., et al., 1988, A\&A 203, 399

Morris D., Lamb J., Lazareff B., et al., 1996, Phase-Coherent Holography of the $30 \mathrm{~m}$ Telescope in September 1996, IRAM Int. Report

Morris D., Lazareff B., Mattiocco F., et al., 1997, Holography of the $30 \mathrm{~m}$ Telescope in September 1997, IRAM Int. Report
Prestage R., 1993, The JCMT Newslett. July 27

Robieux J., 1966, Ann. Radioelectr. 11, 633

Rush W.V.T., Wohlleben R., 1982, IEEE Trans. Ant. Propag. AP-30, 784

Ruze J., 1952, Suppl. al Nuovo Cimento 9, 364

Ruze J., 1966, Proc. IEEE 54, 633

Scheffler H., 1962, Z. f. Astrophys. 55, 1

Schwesinger G., 1972, Optik 34, 553

Shifrin Y.S., 1971, Statistical Antenna Theory. The Golem Press, Boulder, Colorado, U.S.A.

Smith W.T., Bastian R.J., 1997, IEEE Trans. Ant. Propag. $\mathrm{AP}-45,5$

Väisälä Y., 1922, Ann. Univ. Finnicae Aboensis, Turku, Ser A 1, No. 2

Vu T.B., 1969, Proc. IEE 116, 195

Vu T.B., 1970a, Int. J. Electron. 29, 165

Whyborn N.D., Morris D., 1995, Experim. Astron. 6, 43 